\title{
Significance of Cardiac Rehabilitation on Visit-to-Visit Variability of Blood Pressure in Patients With Cardiovascular Disease in a 12-Month Follow-Up
}

\author{
Toshihisa Ishida ${ }^{\text {a }}$, Shin-ichiro Miura, b, d, Kanta Fujimia, c, Makito Futamia ${ }^{\text {a }}$, Yoko Ueda ${ }^{\text {a }}$, \\ Takashi Ueda ${ }^{a}$, Tadaaki Arimura ${ }^{a}$, Rie Koyoshi ${ }^{a}$, Yuhei Shiga ${ }^{a}$, Ken Kitajima ${ }^{a}$, Keijiro Saku ${ }^{\text {a b }}$
}

\begin{abstract}
Background: Visit-to-visit variability (VVV) in blood pressure (BP) has been shown to be a strong predictor of cardiovascular disease (CVD). However, the long-term effect of comprehensive cardiac rehabilitation (CR) with exercise training on VVV in BP has not yet been established. Therefore, we evaluated the long-term effects of CR on VVV in BP in patients with CVD.

Methods: Twenty-two CVD patients in a 12-month CR program who had at least six clinic visits per month to measure BP were enrolled. We determined VVV in BP expressed as the standard deviation of average BP every month for 12 months.

Results: The mean age was $70 \pm 8$ years and the body mass index was $24.4 \pm 4.9 \mathrm{~kg} / \mathrm{m}^{2}$. In addition, the percentage $(\%)$ of males, $\%$ heart failure and $\%$ ischemic heart disease were $77 \%, 55 \%$ and $27 \%$, respectively. Patients who had uncontrolled BP at baseline showed a significant reduction of both systolic BP (SBP) and diastolic BP (DBP). VVV in SBP in the first month was significantly less than that in the last month, although there was no difference in VVV in DBP. Patients were divided into larger (L-) and smaller (S-) VVV in SBP groups according to the average value of VVV in SBP as a cut-off. The L-VVV in SBP group, but not the S-VVV in SBP group, showed a significant reduction of VVV in SBP.
\end{abstract}

Conclusion: Comprehensive CR may improve VVV in SBP in CVD patients who have larger VVV in SBP.

Keywords: Exercise training; Cardiac rehabilitation; Blood pressure; Visit-to-visit variability

Manuscript accepted for publication January 12, 2017

aDepartment of Cardiology, Fukuoka University School of Medicine, Fukuoka, Japan

bepartment of Molecular Cardiovascular Therapeutics, Fukuoka University School of Medicine, Fukuoka, Japan

${ }^{\mathrm{c}}$ Department of Rehabilitation, Fukuoka University Hospital, Fukuoka, Japan

${ }^{\mathrm{d} C o r r e s p o n d i n g ~ A u t h o r: ~ S h i n-i c h i r o ~ M i u r a, ~ D e p a r t m e n t ~ o f ~ C a r d i o l o g y, ~}$

Fukuoka University School of Medicine, Fukuoka, Japan.

Emailmiuras@cis.fukuoka-u.ac.jp

doi: https://doi.org/10.14740/jocmr2915w

\section{Introduction}

Hypertension (HTN) is a major risk factor for the onset and progression of cardiovascular disease (CVD) [1]. When patients have multiple coronary risk factors, the Japanese Society of Hypertension Guidelines for the Management of Hypertension recommend a lower blood pressure (BP) level $(<130 / 80 \mathrm{~mm}$ $\mathrm{Hg}$ ) in the absence of significant coronary artery stenosis in patients with CVD [1]. Although long-term optimal mean BP control is important for preventing the development of CVD, visitto-visit variability (VVV) in BP has been shown to be a strong predictor of CVD and stroke independent of mean BP and other $\mathrm{CV}$ risk factors $[2,3]$. Muntner et al also reported that higher VVV of systolic BP (SBP) is associated with an increased risk for CVD and mortality in a prospective cohort study [4].

Comprehensive cardiac rehabilitation (CR) with exercise training has been shown to improve the prognosis in addition to cardiac function and exercise capacity in patients with CVD $[5,6]$. We previously reported that exercise training significantly reduced BP in patients with HTN through activation of the kinin-kallikrein and dopamine systems and sympathetic inactivation $[7,8]$.

To prevent the repeat occurrence and progression of CVD, it may be important to manage optimal VVV in BP in addition to $\mathrm{BP}$ control in patients with CVD through CR. Although we previously reported that a 3-month CR program improved VVV in BP [9], the long-term effect of CR on VVV in BP has not yet been established. In addition, it remains unclear whether CR induces a BP reduction in patients with CVD over a long term. Accordingly, the aim of this study was to determine whether comprehensive CR improves VVV in BP and induces a BP reduction in patients with CVD in a 12 -month CR program.

\section{Methods}

\section{Study population and protocol}

Twenty-two CVD patients who had ischemic heart disease (IHD), heart failure (HF), sick sinus syndrome, pulmonary embolism and/or cardiovascular surgery and who participated in a 12-month CR program and had at least six clinic visits per month to measure BP were retrospectively enrolled. All data 
Table 1. Patient Characteristics and Biochemical Parameters in Blood at Baseline and After 12 Months in All Patients $(n=22)$

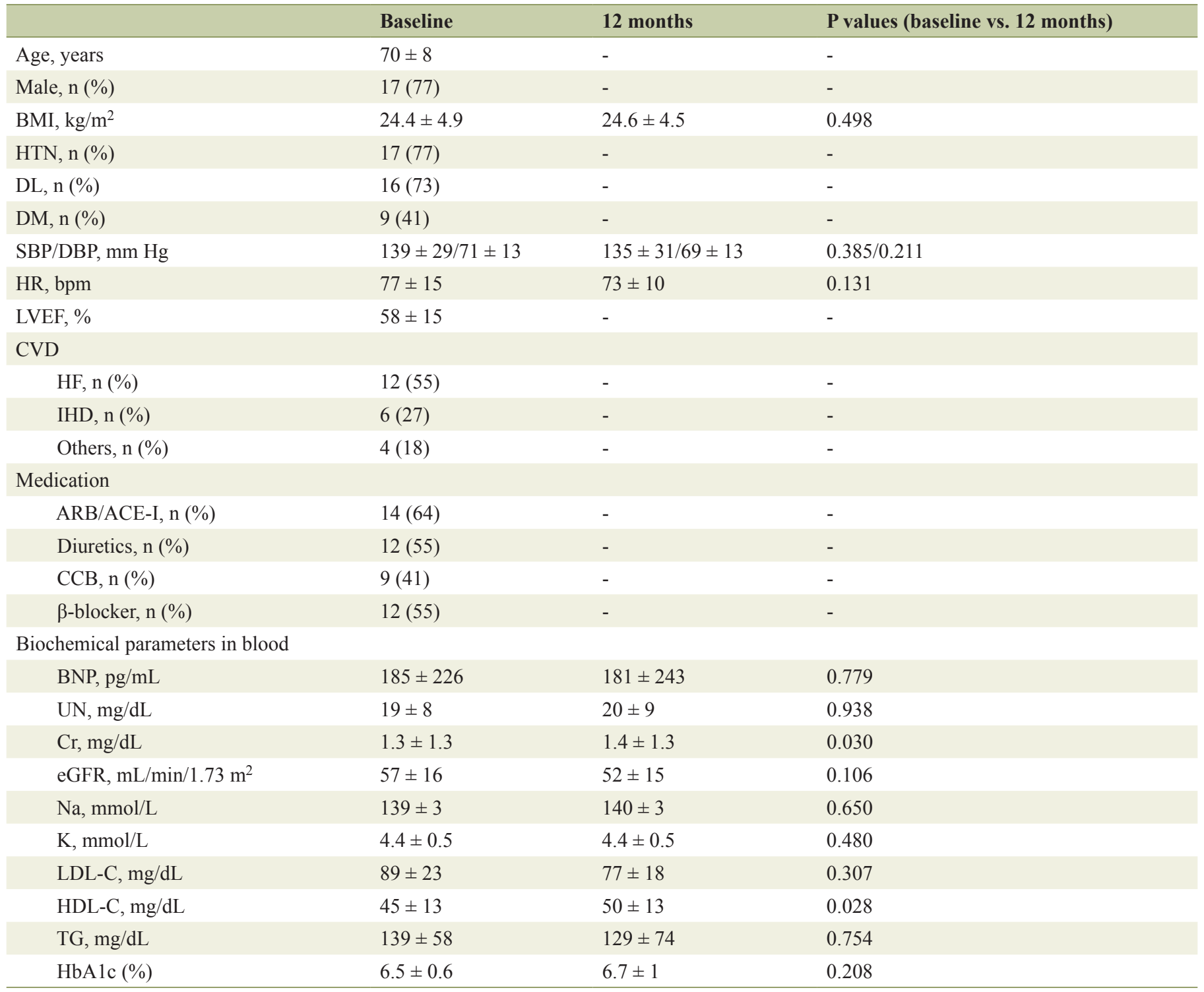

BMI: body mass index; HTN: hypertension; DL: dyslipidemia; DM: diabetes mellitus; SBP: systolic blood pressure; DBP: diastolic blood pressure; HR: heart rate; LVEF: left ventricular ejection fraction; CVD: cardiovascular disease; HF: heart failure; IHD: ischemic heart disease; ARB: angiotensin II receptor blocker; ACE-I: angiotensin converting enzyme inhibitor; CCB: calcium channel blocker; BNP: brain natriuretic peptide; UN: urea nitrogen; Cr: creatinine; eGFR: estimated glomerular filtration rate; Na: sodium; K: potassium; LDL-C: low-density lipoprotein cholesterol; HDL-C: high-density lipoprotein cholesterol; TG: triglyceride; HbA1c: hemoglobin A1c.

were collected at baseline and after 12 months. The study was approved by the ethics committee of Fukuoka University Hospital (\#15-5-09). We retrospectively collected and analyzed all data using the database of Fukuoka University Hospital.

\section{Exercise protocol}

The CR group participated in a supervised exercise training program at the hospital's gym at least six times a month for 12 months. The exercise intensity was chosen to be the estimated $50 \%$ of peak $\mathrm{VO}_{2}$ according to heart rate (HR) and Borg's scale 11 - 13 during exercise, as described elsewhere $[5,10]$. Each session lasted about $1 \mathrm{~h}$, beginning with a warm-up exercise for $10 \mathrm{~min}$, followed by $30 \mathrm{~min}$ of cycling or walking at the indicated exercise intensity and $20 \mathrm{~min}$ of cooling down and stretching. BP and HR were measured at rest and at the end of exercise, and an electrocardiogram (Central Monitor (DS-5700), Fukuda Denshi Co. Ltd., Tokyo, Japan) and Borg's scale were recorded during exercise. All patients were routinely screened before each exercise session, such as by symptoms, HR and rhythm, electrocardiogram, BP and medication regimen. The following conditions had to be managed during exercise: angina, dysrhythmia, hypotension, HTN, 

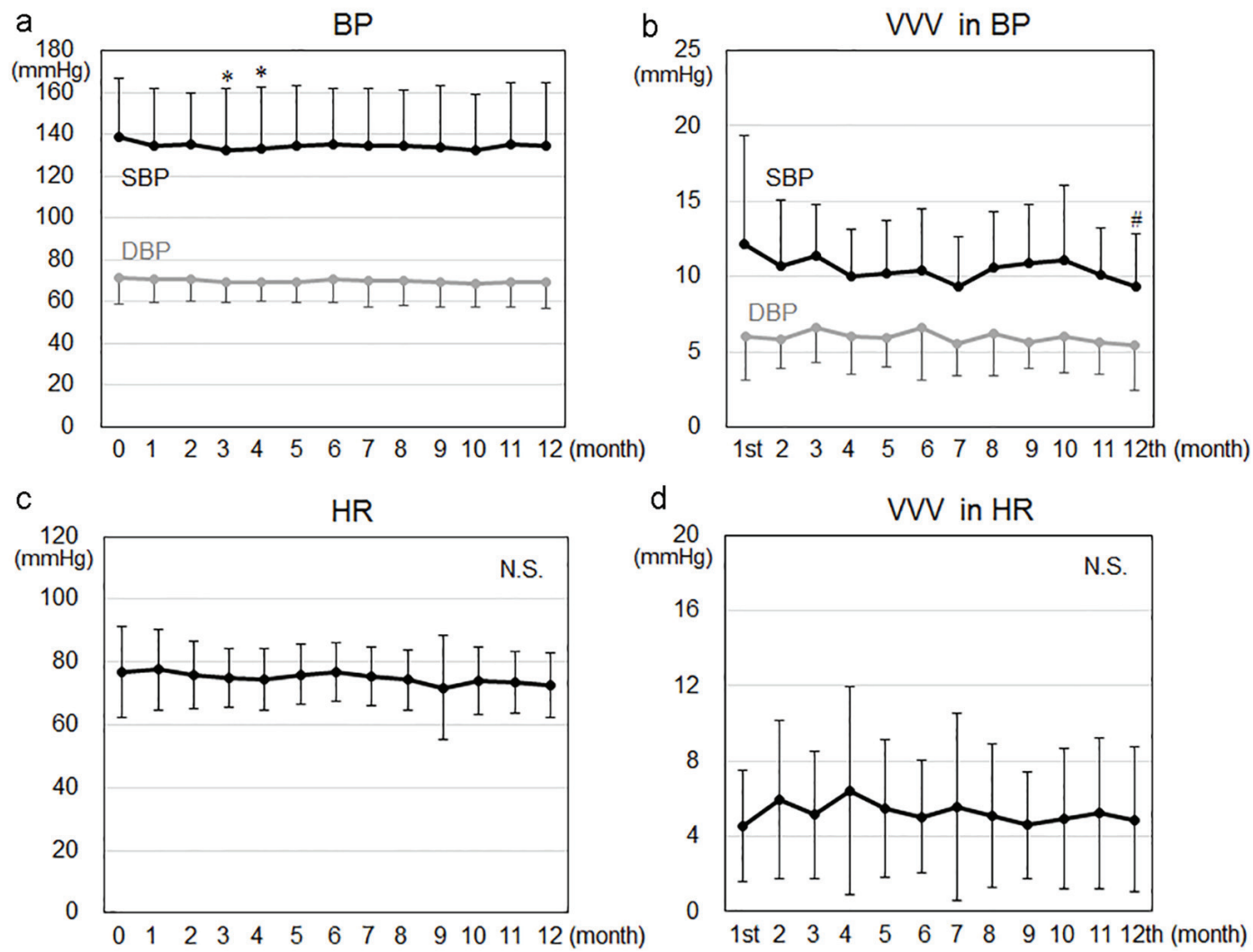

Figure 1. Time courses of changes in blood pressure (BP) (a), visit-to-visit variability (VVV) in BP (b), heart rate (HR) (c) and VVV in HR (d) in all patients. ${ }^{*} \mathrm{P}<0.05$ vs. 0 months. \#P < 0.05 vs. 1st month. SBP: systolic blood pressure; DBP: diastolic blood pressure; N.S.: not significant.

dyspnea, decreased exercise tolerance and cardiac or respiratory arrest.

\section{Measurement of BP and assessment of BP parameters}

BP was determined using an automated sphygmomanometer (HEM-8723, Omron, Tokyo, Japan) after at least 5 min of rest. SBP and diastolic BP (DBP) measurements were obtained at every visit for CR for 12 months. We used the data from six clinic visits for each of the 12 months and determined VVV in both SBP and DBP and in HR expressed as a standard deviation (SD) of average SBP, average DBP and average HR.

\section{Data collection}

Fasting blood samples were collected at baseline and after 12 months. Patient characteristics, including medications at baseline and brain natriuretic peptide (BNP), urea nitrogen (UN), creatinine $(\mathrm{Cr})$, estimated glomerular filtration rate (eGFR), sodium $(\mathrm{Na})$, potassium $(\mathrm{K})$, low-density lipoprotein cholesterol (LDL-C), high-density lipoprotein cholesterol (HDL-C), triglyceride (TG) and hemoglobinAlc (HbAlc) in blood, were assessed at baseline and after 12 months. IHD was defined as lumen diameter stenosis $>50 \%$ in at least one major coronary artery as determined by coronary angiography and as diagnosed by old myocardial infarction. HF was assumed based on the medical history including medications and cardiac function.

\section{Statistical analysis}

A statistical analysis was performed using the Stat View statistical software package (Stat View 5; SAS Institute INC., Cary, $\mathrm{NC})$. Data were expressed as the mean $\pm \mathrm{SD}$ or number $(\%)$. The significance of differences was evaluated using Wilcoxon signed-rank test or Student's $t$-test for continuous variables and the $\chi^{2}$ test for categorical variables. A value of $\mathrm{P}<0.05$ was considered significant.

\section{Results}

\section{Patient characteristics at baseline in all patients}

The patient characteristics at baseline are shown in Table 1. The mean age was $70 \pm 8$ years and the body mass index was $24.4 \pm 4.9 \mathrm{~kg} / \mathrm{m}^{2}$. In addition, the percentage (\%) of males, $\%$ HF and $\%$ IHD were $77 \%, 55 \%$ and $27 \%$, respectively. The use 
Table 2. Patient Characteristics at Baseline and After 12 Months in the Controlled and Uncontrolled BP Groups

\begin{tabular}{|c|c|c|c|c|}
\hline & \multicolumn{2}{|c|}{ Controlled BP group $(n=10)$} & \multicolumn{2}{|c|}{ Uncontrolled BP group $(n=12)$} \\
\hline & Baseline & 12 months & Baseline & 12 months \\
\hline Age, years & $70 \pm 8$ & - & $71 \pm 9$ & - \\
\hline BMI, kg & $24.2 \pm 3.4$ & $24.6 \pm 3.3$ & $24.5 \pm 6.1$ & $24.7 \pm 5.5$ \\
\hline HTN, n (\%) & $8(80)$ & - & $9(75)$ & - \\
\hline SBP/DBP, mm Hg & $115 \pm 15 / 63 \pm 11$ & $118 \pm 22 / 65 \pm 11$ & $158 \pm 21 * * / 79 \pm 9 * *$ & $149 \pm 30 / 73 \pm 13 *$ \\
\hline $\mathrm{HR}, \mathrm{bpm}$ & $80 \pm 17$ & $71 \pm 11$ & $74 \pm 13$ & $74 \pm 10$ \\
\hline LVEF, \% & $57 \pm 17$ & & $60 \pm 13$ & \\
\hline \multicolumn{5}{|l|}{ CVD } \\
\hline \multicolumn{5}{|l|}{ Medication } \\
\hline ARB/ACE-I, n (\%) & $8(80)$ & - & $6(50)$ & - \\
\hline Diuretics, n (\%) & $3(30)$ & - & $6(50)$ & - \\
\hline $\mathrm{CCB}, \mathrm{n}(\%)$ & $7(70)$ & - & $5(42)$ & - \\
\hline$\beta$-blocker, n (\%) & $6(60)$ & - & $6(50)$ & - \\
\hline
\end{tabular}

BMI: body mass index; HTN: hypertension; DL: dyslipidemia; DM: diabetes mellitus; SBP: systolic blood pressure; DBP: diastolic blood pressure; HR: heart rate; LVEF: left ventricular ejection fraction; CVD: cardiovascular disease; HF: heart failure; IHD: ischemic heart disease; ARB: angiotensin II receptor blocker; ACE-I: angiotensin converting enzyme inhibitor; CCB: calcium channel blocker. ${ }^{*} \mathrm{P}<0.05$ vs. at baseline in the uncontrolled group. ${ }^{* *} \mathrm{P}<0.01$ vs. at baseline in the controlled group.

of angiotensin II receptor blocker/angiotensin converting enzyme inhibitor, diuretics, calcium channel blocker (CCB) and $\beta$-blocker were $64 \%, 55 \%, 41 \%$ and $55 \%$, respectively.

\section{Biochemical parameters in blood between baseline and af- ter 12 months in all patients}

Next, we assessed changes in biochemical parameters in blood between baseline and after 12 months (Table 1). There were no significant changes in BNP, UN, eGFR or K in blood between baseline and after 12 months. The levels of HDL-C and $\mathrm{Cr}$ showed a significant increase between baseline and after 12 months, whereas there were no changes in LDL-C, TG or eGFR between baseline and after 12 months (Table 1).

\section{Time course of changes in levels of BP and VVV in BP in all patients}

Figure 1 shows the time course of changes in levels of BP and VVV in BP in all patients. Although there were significant time-course changes in SBP after 3 and 4 months of the CR program, there were no significant changes in SBP or DBP between 0 and 12 months. While VVV in SBP in the first month (12.1 $\mathrm{mm} \mathrm{Hg}$ ) was significantly lower than that in the last (12th) month $(9.3 \mathrm{~mm} \mathrm{Hg})$, there was no difference in VVV in DBP between the first and 12th months. There were no significant time-course changes in HR and VVV in HR over the 12 months.

\section{Patient characteristics at baseline and after 12 months in controlled and uncontrolled BP groups}

The patients were divided into controlled $(\mathrm{n}=10, \mathrm{SBP} / \mathrm{DBP}$ $<140 / 90 \mathrm{~mm} \mathrm{Hg}$ at baseline) and uncontrolled $(\mathrm{n}=12, \mathrm{SBP} /$ $\mathrm{DBP} \geq 140 / 90 \mathrm{~mm} \mathrm{Hg}$ at baseline) BP groups (Table 2). There were no differences in the patient characteristics at baseline or after 12 months between the uncontrolled and controlled BP groups, except that SBP and DBP at baseline in the uncontrolled BP group were significantly higher than those in the controlled BP group.

Time course of changes in levels of SBP and DBP in the controlled and uncontrolled BP groups

In the uncontrolled BP groups, SBP at baseline was significantly decreased after 2, 3, 4, 5, 7, 8 and 10 months (Fig. 2). In addition, DBP at baseline in the uncontrolled BP group was significantly decreased after $2,3,4,7,8,9,10,11$ and 12 

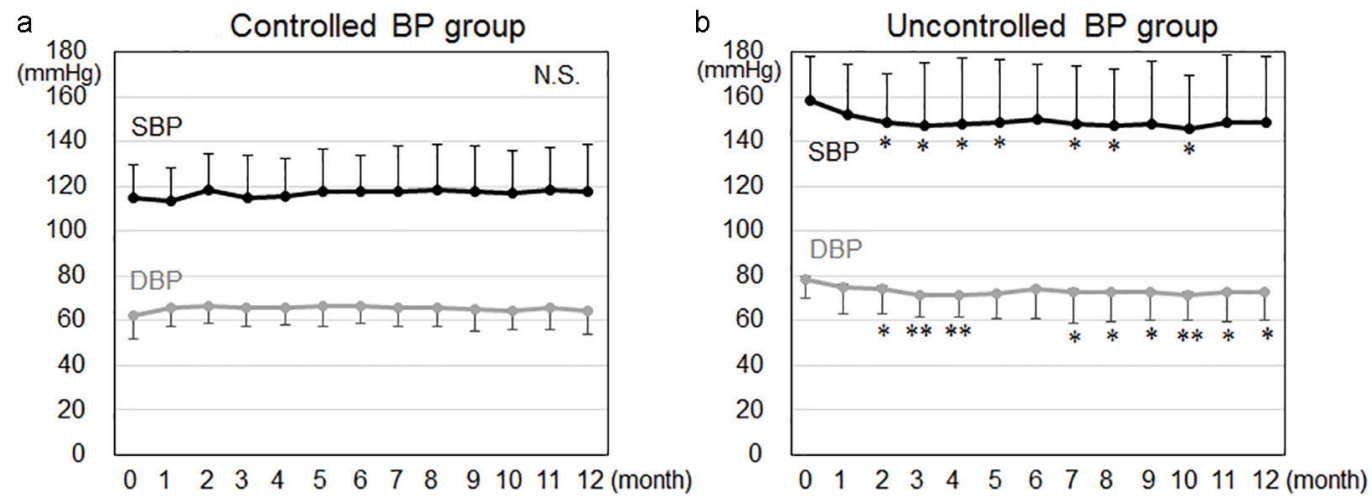

Figure 2. Time courses of changes in blood pressure (BP) in the controlled BP (a) and uncontrolled BP (b) groups. ${ }^{*} P<0.05$, ${ }^{* *} P$ $<0.01$ vs. 0 months. SBP: systolic blood pressure; DBP: diastolic blood pressure; N.S.: not significant.

months. On the other hand, there were no time-course changes in SBP or DBP in the controlled BP group.

\section{Patient characteristics at baseline and after 12 months in the larger (L-) and smaller (S-) VVV in SBP groups}

The patients were divided into two groups according to the average value of VVV in SBP as a cut-off: L-VVV $(n=6)$ and S-VVV $(n=16)$ in SBP groups (Table 3$)$. There were no differences in patient characteristics including SBP $(153 \pm 25$ $\mathrm{mm} \mathrm{Hg}$ (L-VVV in SBP group) vs. $133 \pm 28$ (S-VVV in SBP group $), \mathrm{P}=0.145)$ and $\mathrm{DBP}(78 \pm 13$ vs. $69 \pm 12(\mathrm{P}=0.174))$ at baseline between the L-VVV and S-VVV in SBP groups. In addition, there were no significant changes in SBP or DBP between baseline and 12 months in both the L-VVV and S-VVV

Table 3. Patient Characteristics and Biochemical Parameters in Blood at Baseline and After 12 Months in the Smaller (S-) and Larger (L-) VVV in SBP Groups

\begin{tabular}{|c|c|c|c|c|}
\hline & S-VVV & $\operatorname{group}(n=16)$ & L-VV & $P \operatorname{group}(n=6)$ \\
\hline & Baseline & 12 months & Baseline & 12 months \\
\hline Age, years & $72 \pm 7$ & - & $66 \pm 10$ & - \\
\hline BMI, kg & $24.9 \pm 5.2$ & $25.0 \pm 4.5$ & $23.0 \pm 4.1$ & $23.6 \pm 4.9$ \\
\hline HTN, n (\%) & $13(81)$ & - & $4(67)$ & - \\
\hline DM, n (\%) & $6(38)$ & - & $3(50)$ & - \\
\hline $\mathrm{SBP} / \mathrm{DBP}, \mathrm{mm} \mathrm{Hg}$ & $133 \pm 28 / 69 \pm 12$ & $128 \pm 27 / 67 \pm 11$ & $153 \pm 25 / 78 \pm 13$ & $153 \pm 35 / 75 \pm 15$ \\
\hline HR, bpm & $77 \pm 16$ & $72 \pm 9$ & $78 \pm 10$ & $75 \pm 13$ \\
\hline LVEF, \% & $57 \pm 16$ & - & $63 \pm 9$ & - \\
\hline Others, n (\%) & $3(19)$ & - & $1(17)$ & - \\
\hline Medication & & & & \\
\hline ARB/ACE-I, n (\%) & $11(69)$ & - & $3(50)$ & - \\
\hline Diuretics, n (\%) & $9(56)$ & - & $3(50)$ & - \\
\hline $\mathrm{CCB}, \mathrm{n}(\%)$ & $6(38)$ & - & $3(50)$ & - \\
\hline$\beta$-blocker, n (\%) & $10(63)$ & - & $2(33)$ & - \\
\hline
\end{tabular}

VVV: visit-to-visit variability; BMI: body mass index; HTN: hypertension; DL: dyslipidemia; DM: diabetes mellitus; SBP: systolic blood pressure; DBP: diastolic blood pressure; HR: heart rate; LVEF: left ventricular ejection fraction; CVD: cardiovascular disease; HF: heart failure; IHD: ischemic heart disease; ARB: angiotensin II receptor blocker; ACE-I: angiotensin converting enzyme inhibitor; CCB: calcium channel blocker. 
Table 4. Patient Characteristics at Baseline and After 12 Months in the Smaller (S-) and Larger (L-) VVV in DBP Groups

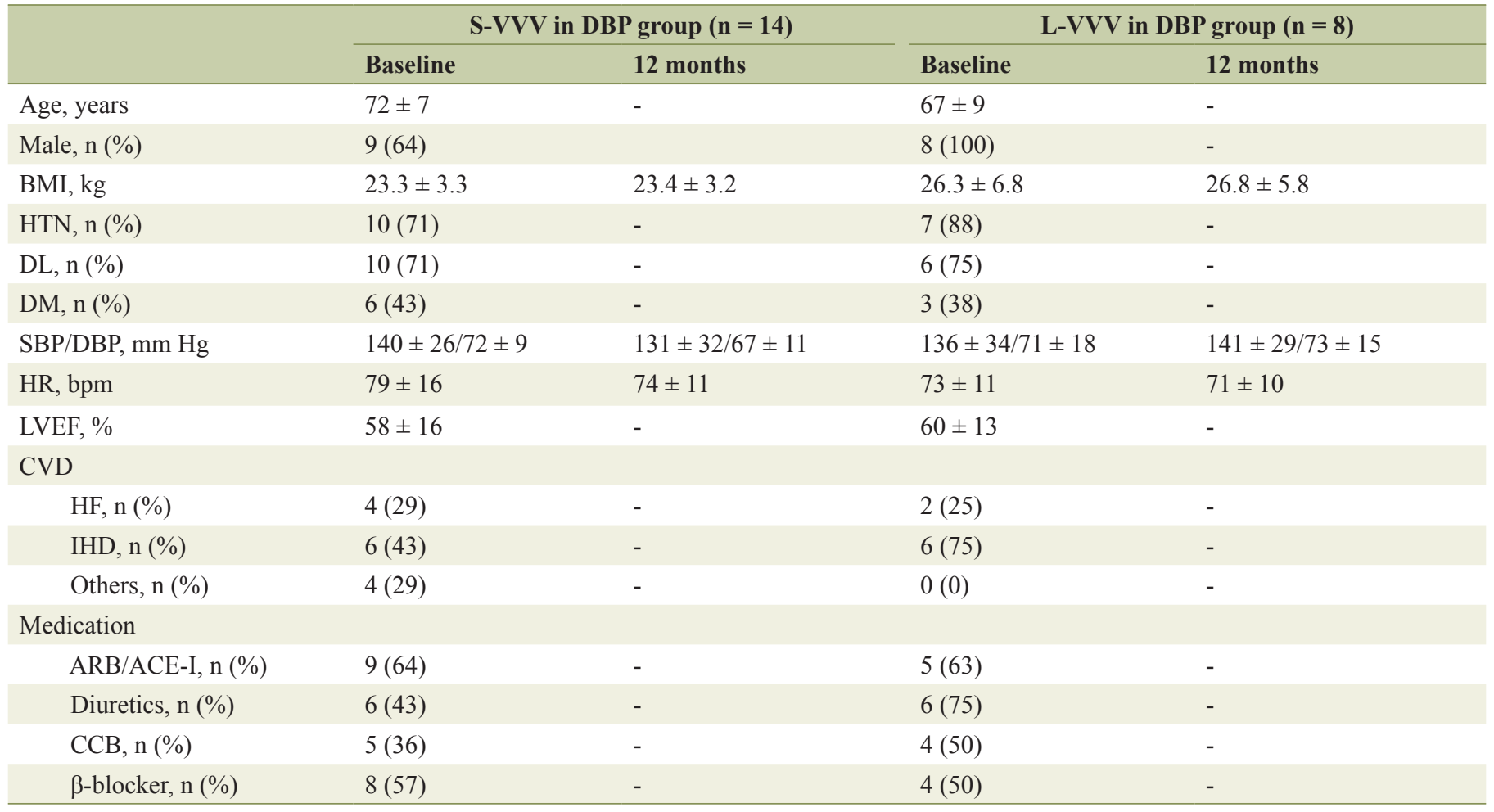

VVV: visit-to-visit variability; BMI: body mass index; HTN: hypertension; DL: dyslipidemia; DM: diabetes mellitus; SBP: systolic blood pressure; DBP: diastolic blood pressure; HR: heart rate; LVEF: left ventricular ejection fraction; CVD: cardiovascular disease; HF: heart failure; IHD: ischemic heart disease; ARB: angiotensin II receptor blocker; ACE-I: angiotensin converting enzyme inhibitor; CCB: calcium channel blocker.

in SBP groups.

\section{Patient characteristics at baseline and after 12 months in the L-VVV and S-VVV in DBP groups}

Next, we divided the patients into two groups according to the average value of VVV in DBP as a cut-off: L-VVV (n $=8)$ and S-VVV $(n=14)$ in DBP groups (Table 4). Again, there were no differences in patient characteristics at baseline and after 12 months between the L-VVV and S-VVV in DBP groups.

\section{Time courses of changes in VVV in BP in the L-VVV and $\mathrm{S}-\mathrm{VVV}$ in BP groups}

Figure 3 shows the time courses of changes in VVV in BP in both the L-VVV and S-VVV in BP groups. As shown in Figure 3a, VVV in SBP in the first month was significantly higher than those in the second, fourth, fifth, sixth, seventh, eighth, ninth, 11th and 12th months in the L-VVV in SBP group, and significantly lower than those in the third and eighth months in the S-VVV in SBP group. In addition, VVV in DBP in the first month was significantly higher than those in the second, fifth, seventh, eighth, ninth, 10th and 11th months in the L-VVV in DBP group, and significantly lower than that in the third month in the S-VVV in DBP group (Fig. 3b).

\section{Discussion}

This study had two major findings. First, a 12-month CR program improved VVV in BP in patients with L-VVV in BP at baseline. Second, the CR program reduced BP in patients who had $\mathrm{BP} \geq 140 / 90 \mathrm{~mm} \mathrm{Hg}$ at baseline.

We found that a 12 -month CR program improved VVV in BP. The mechanisms of this improvement in VVV in BP with CR are not yet clear. Generally, it is considered that an increased variability of BP may be associated with atrial stiffness [11] and abnormal autonomic function [12]. Exercise training has been associated with the suppression of sympathetic nerve activity in patients with HTN $[8,13]$. Such training has been shown to prevent deterioration of the arterial baroreflex control of sympathetic nerve activity in chronic HF patients [14]. In addition, a 20-week CR program was associated with an improvement in arterial stiffness [15], although it is still controversial whether CR improves atrial stiffness. A proposed mechanism is that $\mathrm{CR}$ with exercise training may improve atrial stiffness and reduce excessive sympathetic nerve activity in patients with CVD. Although these mechanisms should be considered in the present study, we need to confirm this observation by analyzing atrial stiffness and sympathetic nerve activity. The patients in this study had HF and IHD. Since CR 

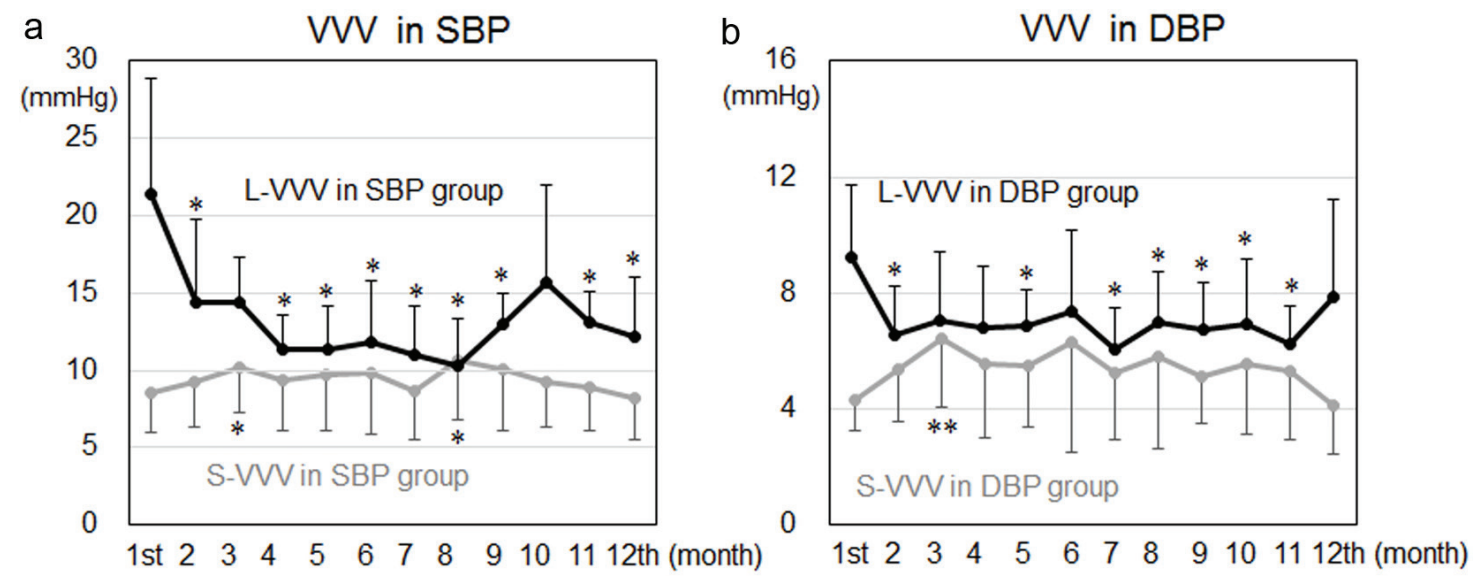

Figure 3. Time courses of changes in visit-to-visit variability (VVV) in systolic blood pressure (SBP) in the larger (L-) and smaller (S-) VVV in SBP groups (a) and diastolic BP (DBP) in the L-VVV and S-VVV in DBP groups (b). ${ }^{*} P<0.05,{ }^{* *} P<0.01$ vs. 1 st month.

is known to improve coronary risk factors and the prognosis in patients with IHD [6] as well as cardiac function in HF patients [8], the present study provides evidence that patients with IHD and/or HF may benefit from CR, probably due to an improvement of VVV in BP.

In this study, 12 of the 22 patients changed the doses and/ or kinds of anti-hypertensive medications during the study period. Although we do not know whether each antihypertensive drug affects the variability in BP to the same extent, the variability in BP is reduced by CCBs, reduced slightly less by diuretics, and increased by $\beta$-blockers, which explains the presence of class differences in the effects of drugs on stroke risk in randomized controlled trials $[16,17]$. CCBs have been shown to reduce the interindividual variability in SBP when used in combination with another agent, and a smaller reduction is seen with diuretics [18]. It is also important to better understand the dose-response effects of each anti-hypertensive drug. Higher doses of CCBs have been shown to reduce SBP variability [18]. Although the class differences and dose-response effects of anti-hypertensive drugs may influence VVV in BP, we found that a 12-month CR program improved VVV in BP.

In this study, CR significantly decreased BP in patients who had $\mathrm{BP} \geq 140 / 90 \mathrm{~mm} \mathrm{Hg}$ at baseline, although there were no significant changes in BP between baseline and 12 months in all patients. This is a reasonable finding because patients who had $\mathrm{BP}<140 / 90 \mathrm{~mm} \mathrm{Hg}$ at baseline did not need to decrease their BP. A decrease in BP with exercise training is a well-recognized phenomenon in patients with chronic HF and HTN [7, $8,13,19]$. Exercise training is also recommended to lower BP in patients with HF [20]. In addition, aerobic exercise promotes a decrease in percentage body fat, decreases TG levels, and increases HDL cholesterol while reducing BP [21, 22].

The present study has several limitations. First, it was retrospective and from a single center with a relatively small sample size. Second, we did not confirm an improvement in atrial stiffness or sympathetic nerve activity. Third, each patient received different doses and kinds of medications. Therefore, a large study is needed to confirm that CR improves VVV in BP.

In conclusion, comprehensive $\mathrm{CR}$ program may improve
VVV in SBP in CVD patients who have a larger VVV in SBP.

\section{Conflicts of Interest}

KS is a Chief Director and SM is a Director of NPO Clinical and Applied Science, Fukuoka, Japan. KS has an Endowed Department of "Department of Molecular Cardiovascular Therapeutics" supported by MSD, Co. LTD. SM belongs to the Department of Molecular Cardiovascular Therapeutics supported by MSD, Co. LTD.

\section{References}

1. Shimamoto K, Ando K, Fujita T, Hasebe N, Higaki J, Horiuchi $\mathrm{M}$, Imai $\mathrm{Y}$, et al. The Japanese Society of Hypertension Guidelines for the Management of Hypertension (JSH 2014). Hypertens Res. 2014;37(4):253-390.

2. Rothwell PM, Howard SC, Dolan E, O'Brien E, Dobson JE, Dahlof B, Sever PS, et al. Prognostic significance of visit-to-visit variability, maximum systolic blood pressure, and episodic hypertension. Lancet. 2010;375(9718):895905.

3. Diaz KM, Tanner RM, Falzon L, Levitan EB, Reynolds K, Shimbo D, Muntner P. Visit-to-visit variability of blood pressure and cardiovascular disease and all-cause mortality: a systematic review and meta-analysis. Hypertension. 2014;64(5):965-982.

4. Muntner P, Whittle J, Lynch AI, Colantonio LD, Simpson LM, Einhorn PT, Levitan EB, et al. Visit-to-Visit Variability of Blood Pressure and Coronary Heart Disease, Stroke, Heart Failure, and Mortality: A Cohort Study. Ann Intern Med. 2015;163(5):329-338.

5. Conraads VM, Vanderheyden M, Paelinck B, Verstreken S, Blankoff I, Miljoen H, De Sutter J, et al. The effect of endurance training on exercise capacity following cardiac resynchronization therapy in chronic heart failure patients: a pilot trial. Eur J Cardiovasc Prev Rehabil. 
2007;14(1):99-106.

6. Taylor RS, Brown A, Ebrahim S, Jolliffe J, Noorani H, Rees K, Skidmore B, et al. Exercise-based rehabilitation for patients with coronary heart disease: systematic review and meta-analysis of randomized controlled trials. Am J Med. 2004;116(10):682-692.

7. Miura S, Tashiro E, Sakai T, Koga M, Kinoshita A, Sasaguri M, Ideishi M, et al. Urinary kallikrein activity is increased during the first few weeks of exercise training in essential hypertension. J Hypertens. 1994;12(7):815-823.

8. Arakawa K, Miura S, Koga M, Kinoshita A, Urata H, Kiyonaga A. Activation of renal dopamine system by physical exercise. Hypertens Res. 1995;18(Suppl 1):S73-77.

9. Ishida T, Miura S, Fujimi K, Ueda T, Ueda Y, Matsuda T, Sakamoto M, et al. Visit-to-Visit Variability and Reduction in Blood Pressure After a 3-Month Cardiac Rehabilitation Program in Patients With Cardiovascular Disease. Int Heart J. 2016;57(5):607-614.

10. Belardinelli R, Capestro F, Misiani A, Scipione P, Georgiou D. Moderate exercise training improves functional capacity, quality of life, and endothelium-dependent vasodilation in chronic heart failure patients with implantable cardioverter defibrillators and cardiac resynchronization therapy. Eur J Cardiovasc Prev Rehabil. 2006;13(5):818825.

11. Imai Y, Aihara A, Ohkubo T, Nagai K, Tsuji I, Minami N, Satoh H, et al. Factors that affect blood pressure variability. A community-based study in Ohasama, Japan. Am J Hypertens. 1997;10(11):1281-1289.

12. Palatini P, Julius S. The role of cardiac autonomic function in hypertension and cardiovascular disease. Curr Hypertens Rep. 2009;11(3):199-205.

13. Urata $\mathrm{H}$, Tanabe $\mathrm{Y}$, Kiyonaga $\mathrm{A}$, Ikeda $\mathrm{M}$, Tanaka $\mathrm{H}$, Shindo M, Arakawa K. Antihypertensive and volume-depleting effects of mild exercise on essential hypertension. Hypertension. 1987;9(3):245-252.
14. Groehs RV, Toschi-Dias E, Antunes-Correa LM, Trevizan PF, Rondon MU, Oliveira P, Alves MJ, et al. Exercise training prevents the deterioration in the arterial baroreflex control of sympathetic nerve activity in chronic heart failure patients. Am J Physiol Heart Circ Physiol. 2015;308(9):H1096-1102.

15. Laskey W, Siddiqi S, Wells C, Lueker R. Improvement in arterial stiffness following cardiac rehabilitation. Int $\mathrm{J}$ Cardiol. 2013;167(6):2734-2738.

16. Rothwell PM, Howard SC, Dolan E, O'Brien E, Dobson JE, Dahlof B, Poulter NR, et al. Effects of beta blockers and calcium-channel blockers on within-individual variability in blood pressure and risk of stroke. Lancet Neurol. 2010;9(5):469-480.

17. Webb AJ, Fischer U, Mehta Z, Rothwell PM. Effects of antihypertensive-drug class on interindividual variation in blood pressure and risk of stroke: a systematic review and meta-analysis. Lancet. 2010;375(9718):906-915.

18. Webb AJ, Rothwell PM. Effect of dose and combination of antihypertensives on interindividual blood pressure variability: a systematic review. Stroke. 2011;42(10):28602865.

19. Kokkinos PF, Narayan P, Colleran JA, Pittaras A, Notargiacomo A, Reda D, Papademetriou V. Effects of regular exercise on blood pressure and left ventricular hypertrophy in African-American men with severe hypertension. N Engl J Med. 1995;333(22):1462-1467.

20. The fifth report of the Joint National Committee on Detection, Evaluation, and Treatment of High Blood Pressure (JNC V). Arch Intern Med. 1993;153(2):154-183.

21. Bauman A, Owen N. Habitual physical activity and cardiovascular risk factors. Med J Aust. 1991;154(1):22-28.

22. Ornish D, Brown SE, Scherwitz LW, Billings JH, Armstrong WT, Ports TA, McLanahan SM, et al. Can lifestyle changes reverse coronary heart disease? The Lifestyle Heart Trial. Lancet. 1990;336(8708):129-133. 